\title{
Alternativas de control sobre Bemisia spp. en el cultivo de Capsicum annum L. (criollo) en el municipio de Tepetzintla, Veracruz
}

Alternatives of control on Bemisia spp in Capsicum annum L. (criollo) crop in Tepetzintla, Veracruz

González Acosta Alfredo ${ }^{1 凶}$, González Castro Alfredo ${ }^{1}$, Mateos Rocha Roberto A. ${ }^{1}$, González Cárdenas Julio C. ${ }^{1}$, López Meza Miguel ${ }^{2}$

${ }^{1}$ Facultad de Ciencias Biológicas y Agropecuarias Campus Tuxpan, Universidad Veracruzana, ${ }^{2}$ Facultad de Agronomía, Universidad Autónoma de Sinaloa.

${ }^{\circledR}$ Autor para correspondencia: alfgonzalez@uv.mx

Recibido: 09/07/2015

Aceptado: $14 / 12 / 2015$

\section{RESUMEN}

Para el manejo de Bemisia spp. en cultivo de chile criollo (Capsicum annum L.), se evaluaron diferentes alternativas, entre ellas, los insecticidas químicos: endosulfan, malathion; los bioplaguicidas: Paecilomyces fumosoroseus, Beauveria bassiana; el extracto vegetales: Allium sp.; aceite mineral: Saft-side, los jabones: foca y vel rosita, en ciclo 2008. Se utilizó un diseño de bloques completos al azar con 4 repeticiones, evaluando las poblaciones de adultos, ninfas, y huevos a través de análisis de varianza y una prueba de comparación de medias. Los productos se aplicaron una vez por semana y se hicieron 6 aplicaciones semanales y se tomaron los datos de campo y laboratorio dos días después de cada aplicación. Se observa que los diferentes tratamientos con extractos, aceites, jabones, hongos y químicos los fueron capaces de mantener las poblaciones de adultos de Bemisia spp. en niveles mucho más bajos que el testigo, durante el tiempo que duró el experimento. Las alternativas donde se utilizaron insecticidas químicos, hongos entomopatógenos y los extractos vegetales y aceites minerales fueron similares estadísticamente, y lograron niveles poblacionales de huevos relativamente bajos. En huevos la variante donde se utilizaron los jabones difirió significativamente de todas las demás, que no difirieron entre sí. Ninfas Todas las variantes evaluadas difirieron estadísticamente del testigo, y se observó el menor efecto de regulación de la población de ninfas en la variante con jabones, con diferencia significativa con relación a los demás tratamientos, después, se ubicó la variantes de hongos entomopatógenos, con extractos y aceites, y por último, la de insecticidas químicos que fue el que mayor efecto presento para el control de mosca blanca. Los resultados de este ensayo demuestran que todos los tratamientos difirieron estadísticamente del testigo y que reducen las poblaciones de mosca blanca, por lo que pueden utilizarse exitosamente como alternativa en el manejo integrado de esta plaga.

Palabras clave: Bemisia spp, Capsicum annum, control 


\section{ABSTRACT}

Different alternatives for the management of Bemisia spp. in the "criollo" pepper (Capsicum annum L.) were evaluated during the cycle 2008. Alternatives of control included chemical insecticides endosulfan, malathion; biopesticides Paecilomyces fumosoroseus and Beauveria bassiana; plant extract Allium sp.; mineral oil Saf-t-side; laundry soaps "foca" and "vel Rosita". A randomized complete block design with 4 repetitions was used. Evaluations included adults, nymphs, and egg populations. Data was analyzed by ANOVA and differences between treatments were analyzed by mean comparisons. The different treatments were applied once a week and 6 sprays were conducted. Data were registered two days after each application. Results showed that treatments based on plant extract, mineral oil, laundry soaps, chemical insecticides and biopesticides based on entomopathogenic fungi maintained Bemisia spp. populations in lower leves in comparison with the control treatment. Statistically, similar results were observed on chemical insecticides, entomopathogenic fungi and plant extract, and they showed low levels of egg populations. The effect of laundry soaps showed a distinct effect on egg populations. All the evaluated variables were different statistically in comparison with the control treatment, and a lesser effect on nymphs was observed with the laundry soaps treatment, followed by entomophatogenic fungi, plant extract, and finally the chemical insecticides that showed the best effect to control whiteflies. Results from this experiment showed that all treatments were different in comparison with the control treatment, ant that all of them reduce whiteflies populations. They can be successfully used as an alternative in the integrated management of this insect.

\section{Keywords: Bemisia spp, Capsicum annum, control}

\section{INTRODUCCIÓN}

Las hortalizas son uno de los sectores de la producción agrícola que son autosuficientes. La superficie sembrada de los cultivos en la República Mexicana en 1991 alcanzó la cifra de 860821 ha, de los cuales 71878 ha, fueron cosechadas para generar un volumen de producción de 4584682 toneladas, el chile se encuentra distribuido en todo el mundo y de acuerdo al área sembrada y a los volúmenes de producción, que año con año se incrementan, es actualmente la especia más importante que condimenta los alimentos de la población (FAO, 2004).

El chile, Capsicum annum L. es una de las hortalizas que genera importantes ingresos al país, ya que aproximadamente el $70 \%$ de su producción se exporta a los mercados de estados unidos de América y Canadá; es un cultivo redituable y emplea a miles de trabajadores de las áreas rurales (Arcos et al., 1998).

El cultivo de chile es atacado por una gran cantidad de insectos nocivos que están considerados entre los principales factores limitantes de la producción. Las especies de mosquitas blancas que atacan al cultivo son

Trialeurodes vaporariorum Westwood (Hemiptera: Aleyrodidae), Bemisia tabaci (Gennadius) (Hemiptera: Aleyrodidae), Bemisia argentifolii (Bellows y Perring) (Hemiptera: Aleyrodidae) (Angulo et al., 1994; Alfaro, 1999). Son una de las plagas que más impacto han causado en los últimos años en el mundo (CAB International, 2004). Estos insectos ocasionan en las plantas dos tipos de daños. El directo lo producen al alimentarse de las plantas 
provocando incluso la muerte y decoloración del fruto por efecto de toxinas que transmiten las ninfas, y el indirecto, por ser vectores de más de 60 enfermedades que se presentan en diversos cultivos, principalmente en las hortalizas, y otra forma es por la obstrucción del proceso fotosintético de las plantas al depositar grandes cantidades de mielecilla en el follaje, la cual sirve de sustrato a un complejo de especies de hongos saprofíticos causantes de la "fumagina". En el norte del estado de Sinaloa en 1994, estos insectos causaron pérdidas por casi 10 millones de dólares (Avilés et al., 2004).

En los últimos años, la necesidad de controlar las plagas de insectos en los cultivos, entre ellos el chile, y el interés por minimizar el impacto negativo de los insecticidas sintéticos, ha obligado a buscar nuevas alternativas, basadas en el uso de extractos vegetales y aceites

minerales, hongos entomopatógenos, depredadores, parasitoides, y otros (Rodríguez, 2000; Mareggiani, 2001).

Para este trabajo de investigación los objetivos fueron evaluar alternativas para el manejo de Bemisia spp. mediante la efectividad técnica de diferentes estrategias de medidas biorracionales e insecticidas sintéticos en el cultivo de capsicum anumm l. (criollo). en el municipio de Tepetzintla Veracruz.

\section{MATERIALES Y MÉTODOS}

La investigación se realizó en el rancho "El Estrellal", propiedad del C. Mario Méndez Herbert, en el ejido de corral falso municipio de Tepetzintla Ver. La preparación del terreno, planteo, riego por goteo, fertilización, labores de cultivo, eliminación de malezas, y corte de
González et al., 2015

frutos se realizaron en forma convencional, de la manera acostumbrada por el agricultor. La siembra de chile mirador se realizó en 5 julio del 2008, en bandejas de polietileno de 200 cavidades con el sustrato "peet- moss". A partir de la germinación de la semilla, a las plantas se le hicieron aplicaciones de plaguicidas y fungicidas, durante los 20 días para que estuviera libre de plagas y enfermedades. El trasplante se realizó de manera manual el 9 de agosto del 2008. Cuando la plantas obtuvieron una altura de $15 \mathrm{~cm}$ a $20 \mathrm{~cm}$, con un desarrollo vigoroso y uniforme. Se plantaron en hileras que tuvieron una separación de $85 \mathrm{~cm}$ una de otra y una distancia entre plantas de $30 \mathrm{~cm}$. En el campo se establecieron 20 parcelas que midieron $8 \mathrm{~m}$ de largo por $7 \mathrm{~m}$ de ancho, con una separación entre las mismas de 1.50 m., la superficie por parcela fue de $56 \mathrm{~m}^{2}$. Como parcela útil se disponía de ocho surcos y se tomaron los cuatro centrales de cada unidad experimental para las diferentes evaluaciones.

Los tratamientos consistieron en cinco estrategias diferentes para el control de Bemisia spp.:

1. Aplicaciones con los insecticidas químicos convencionales de mayor uso en la región.

2. Aplicaciones con bioplaguicidas basados en hongos entomopatógenos.

3. Aplicaciones con extractos vegetales y aceites minerales.

4. Aplicación de jabones.

5. Sin aplicaciones de ningún tipo (Testigo).

Estos tratamientos se empezaron aplicar del 27 de septiembre del 2008 y se realizaron semanalmente. Los productos que se utilizaron en las aplicaciones son los que se muestran en la Tabla 1. La selección de uno u otro plaguicida para cada aplicación se basara en la presencia de los organismos nocivos en el cultivo. 
Tabla 1. Productos que se utilizaran como alternativas de control de Bemisia spp.:

\begin{tabular}{lccc}
\hline \multicolumn{1}{c}{ Producto } & $\begin{array}{c}\text { Dosis (i.a) } \\
(\text { g.ha }-1)\end{array}$ & $\begin{array}{c}\text { Dosis (PC) } \\
\left(\text { L.ha }^{-1}\right)\end{array}$ & $\begin{array}{c}\text { No. De } \\
\text { aplicaciones }\end{array}$ \\
\hline Endolsufan (Thiodan 35\% C.E.) & 350 & 1,0 & 4 \\
Malathion (Malathion 500 C.E.) & 500 & 1,0 & 4 \\
P. fumosoroseus (PAE-SIN) & $3,6 \times 10^{12} *$ & 1,5 & 4 \\
B. bassiana (BAE-SIN) & $1,8 \times 10^{12} *$ & 1,5 & 4 \\
Jabón foca & & 2.0 & 4 \\
Vel Rosita & & 2.0 & 4 \\
A. mineral (Saf-T-side) & 800 & 1,0 & 4 \\
E. de Ajo (Biogarlic) & 970 & 1,0 & \\
Testigo & & & 4 \\
\hline Conidios.ha & & & 4 \\
\hline
\end{tabular}

Los experimentos se montaron según un diseño de bloques al azar, con cinco tratamientos y cuatro repeticiones. Se evaluó el efecto de las alternativas utilizadas sobre las poblaciones de las diferentes fases de desarrollo de Bemisia spp. Para la evaluación del efecto de las diferentes alternativas sobre las poblaciones de la mosca blanca se hicieron 6 muestreos con frecuencia semanal, a partir del 29 de septiembre del 2008, 2 días después de la aplicación correspondiente a cada semana, y en el horario de 6:00 a 8:00 am.

En los cuatro surcos centrales de cada parcela (área útil) se descartó $1 \mathrm{~m}$ en sus extremos, quedando 17 plantas para la evaluación por cada surco, 68 en la parcela. De ellas, se seleccionaron al azar seis plantas por surco, realizándose la observación en una hoja del tercio apical de la planta, registrándose el número de adultos presentes. A continuación, dichas hojas fueron cortadas y colocadas en bolsas de plástico para su traslado al laboratorio de la Facultad de Ciencias Biologías Agropecuarias Tuxpan, donde se realizó la cuantificación de huevos y ninfas de la mosca blanca con la ayuda de un microscopio estereoscópico con un aumento de 80X.

Los resultados de las evaluaciones se expresaron como No. de individuos por hoja. Los datos de la población de huevos, ninfas y adultos, para su análisis estadístico se transformaron según la expresión $\mathrm{x}^{1 / 2}$. Para cada una de las fases, con los promedios de las poblaciones de las 6 evaluaciones realizadas en ambos experimentos, se realizaron análisis de varianza, comparándose las medias mediante la Prueba de Tukey, al 5\%. Para el análisis de los datos se utilizó el paquete estadístico del SAS versión 6.12 (Ray, 1982). Además, para cada una de las fases del insecto, para cada ciclo productivo, se elaboraron gráficos de líneas con los valores de la población en los diferentes momentos de evaluación en las distintas alternativas evaluadas. 


\section{RESULTADOS Y DISCUSIONES}

\section{Efecto sobre las poblaciones de Bemisia spp.}

Los análisis de varianza que se realizaron con los datos de la población de adultos, pusieron de manifiesto diferencias altamente significativas entre los tratamientos. En la Figura 1 se muestran los resultados del experimento, al igual que en el caso de los huevos y las ninfas, dentro del ciclo de producción, todas las variantes evaluadas difirieron estadísticamente del testigo, en el cual se alcanzaron poblaciones promedios de más de 40 insectos por hoja.

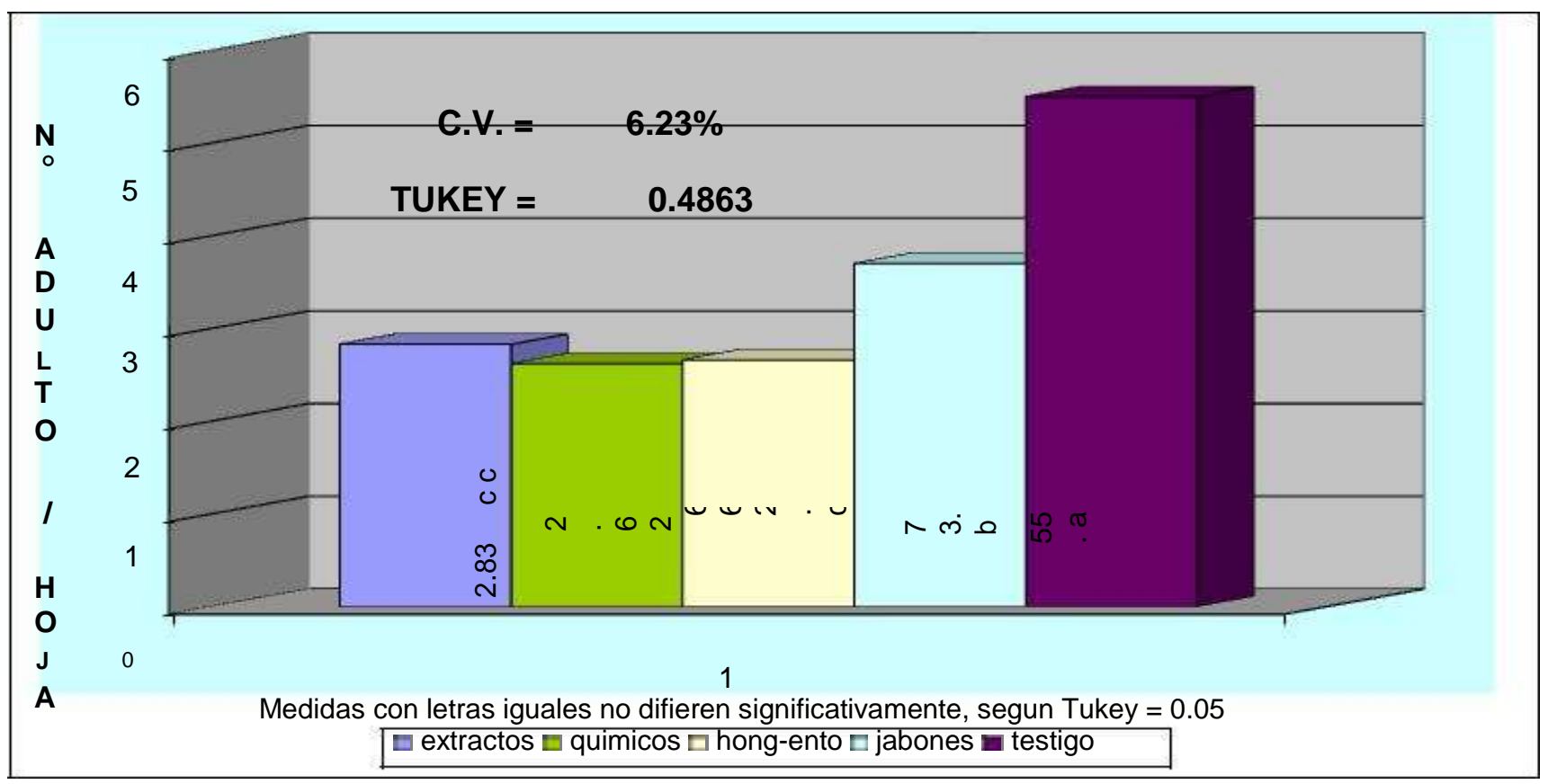

Figura 1. Efectos de las alternativas de manejo sobre los adultos de Bemisia spp. en campo

Las alternativas donde se utilizaron insecticidas químicos, hongos entomopatógenos y los extractos vegetales y aceites minerales fueron similares estadísticamente, y lograron niveles poblacionales de huevos relativamente bajos, por ejemplo, en el caso de los químicos la población estuvo alrededor de los 11 individuos por hoja, o sea, 3.4 veces inferior a la obtenida en el testigo.

Cuando se evaluó el efecto de las diferentes alternativas de manejo sobre las poblaciones de huevos de Bemisia spp. en condiciones de campo, los análisis de varianza efectuados con los datos del promedio de las 6 evaluaciones realizadas, evidenciaron diferencias altamente significativas entre los tratamientos.

Los resultados obtenidos en los experimentos se muestran en la Figura 2, las cuatro alternativas empleadas difirieron estadísticamente del testigo en relación al número de huevos de la mosca blanca por hoja de chile. 


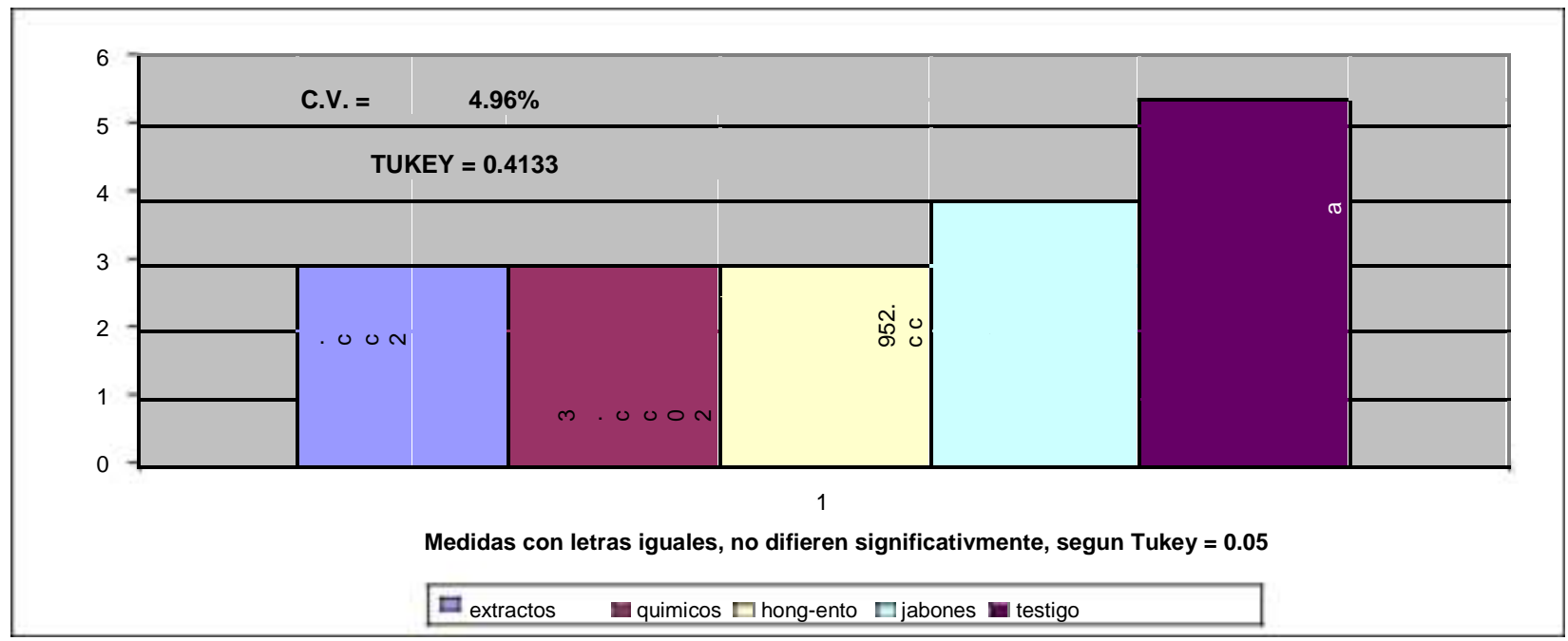

Figura 2. Efectos de las alternativas de manejo sobre huevos de Bemisia spp. en campo

Los análisis estadísticos con los datos de la población de ninfas, también mostraron diferencias significativas entre los tratamientos.
Los resultados obtenidos se muestran en la Figura 3.

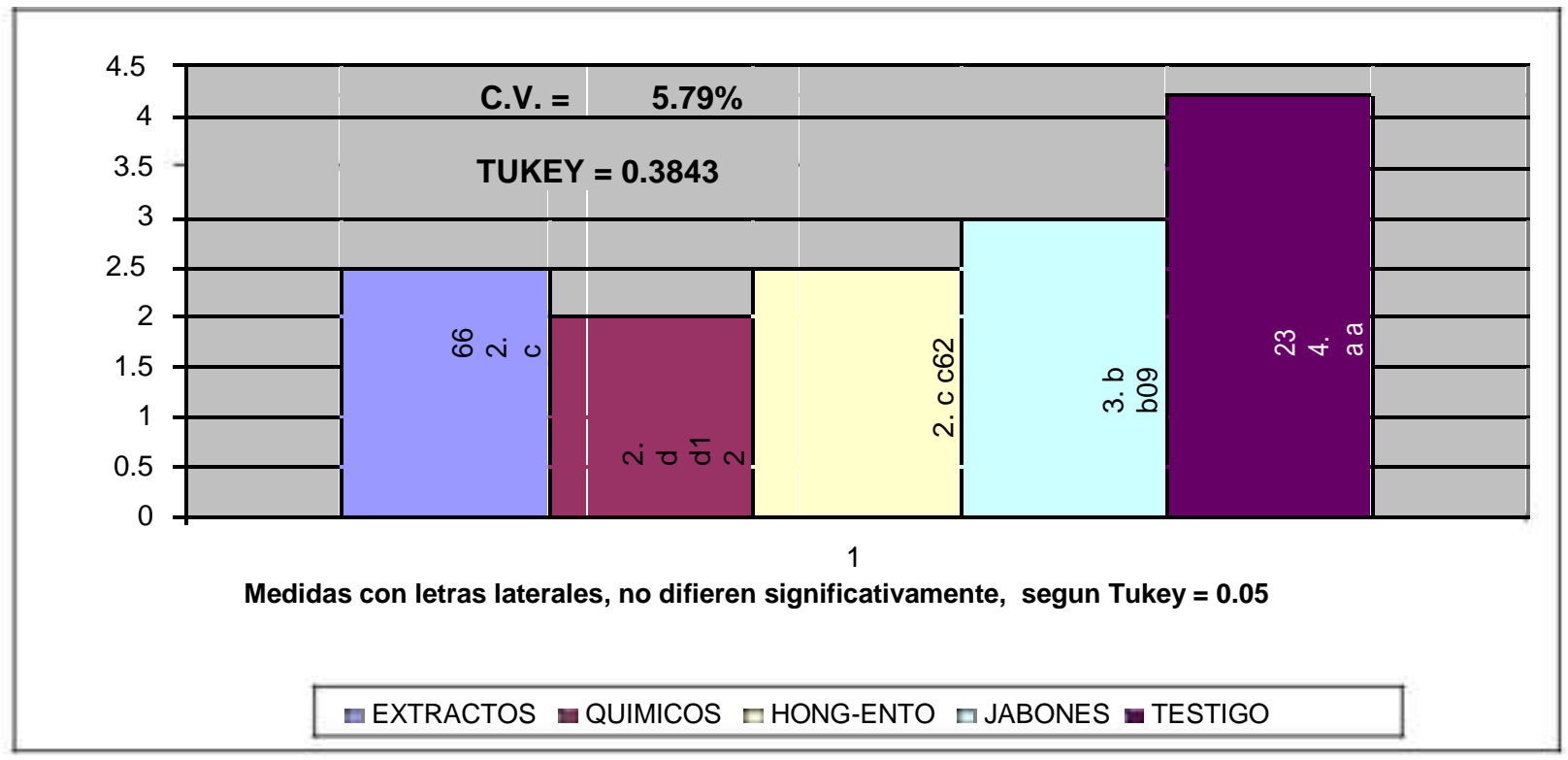

Figura 2. Efectos de las alternativas de manejo sobre huevos de Bemisia spp. en campo

Figura 3. Efectos de las alternativas de manejo sobre ninfas de Bemisia spp. en campo

Todas las variantes evaluadas difirieron estadísticamente del testigo, y se observó el menor efecto de regulación de la población de ninfas en la variante con jabones, con diferencia significativa con relación a los demás tratamientos. A continuación, se ubicó la variantes de hongos entomopatógenos, con extractos y aceites, y por último, la de insecticidas químicos que fue el que mayor efecto presento para el control de mosca blanca.

Estos resultados confirman informes anteriores; en el tratamiento químico se corrobora lo señalado por, López (1998), Valle et al. (2002), y Avilés et al. (2004), quienes han manifestado que el control químico en una de 
las alternativas para el control de Bemisia spp., pero se debe fundamentar en aplicaciones de insecticidas selectivos que respeten en gran medida a los enemigos naturales, y añadieron que la selectividad se puede lograr mediante las características propias del producto, de la formulación así como por la forma y momento de aplicación.

Singh et al. (1981) señalaron que dimetoato y el aceite mineral resultaron muy eficientes en el control de B. tabaci y la reducción de los síntomas virales en frijol. Otros autores (Prabhaker et al., 1998; Arcos et al., 1998; Ruiz y Bolaños, 1999; Bernal, 2003; Borad et al., 2003; Farman et al., 2006) han señalado que los adultos de la mosca blanca son controlados con insecticidas organosintéticos como el endosulfan, el metamidofos y el malathion $\mathrm{y}$ mostraron buena respuesta al efecto acumulativo de los diferentes tratamientos químicos en el tiempo ( 3 d después de la aplicación).

En el caso de las aplicaciones de hongos entomopatógenos se demostró el efecto que pueden tener sobre las poblaciones de huevos, como lo indican Asaff et al. (2003), quienes informaron que $P$. fumosoroseus es un hongo capaz de infectar a la mosquita blanca, Bemisia spp., en todas sus etapas de desarrollo, incluso huevos y causar altos niveles de mortalidad a una velocidad mayor que otros hongos, lo que resulta en un buen control del fitófago en cultivos de invernadero y de campo. Cuando la humedad relativa es alta, el hongo infecta también a los adultos.

García et al. (2000), Poprawski et al. (2000), Orozco et al. (2000), Gindin et al. (2001), Faria et al. (2001), Rodríguez (2003), Ramos et al. (2004), Piron y Lacordaire (2006), han informado que B. bassiana, $P$. fumosoroseus y L. lecanii, ejercen un control eficiente de moscas blancas y presentan un comportamiento muy similar. Al evaluarse su patogenicidad sobre ninfas de Bemisia spp. se comprobó que causaron una mortalidad de 10 a $89 \%$ lo que los convierte en buenos candidatos para el desarrollo de insecticidas biológicos para el control de este fitófago. Manjula et al. (2003) han encontrado que las formulaciones evaluadas de $B$. bassiana en aceite vegetal, no tuvieron un alto efecto sobre los huevos, mientras que los diferentes instares ninfales y los adultos fueron muy sensibles a dichas formulaciones.

El tratamiento de extractos vegetales y aceites minerales reguló las poblaciones de mosca blanca de manera eficiente, resultado este que corrobora los obtenidos por Valderrábano y Uriarte (2001), Silva et al. (2002), Paula y Bleicher (2003) y Aguiar et al. (2003), quienes también trabajaron con extractos de plantas y encontraron un buen efecto de control, y comprobaron una acción antialimentaria y ovicida por parte de los mismos.

El tratamiento de jabones bajo las poblaciones de mosca blanca de acuerdo a los resultados obtenidos coincidiendo Liu y Stansly (1995) estos autores evaluaron el jabón insecticida MPede contra B. argentifolii y encontraron que la mortalidad en huevecillos fue baja debido a que la concentración del $3 \%$ solo causo $25 \%$ de mortalidad pero registraron alta mortalidad de dicho jabón al 2\% para ninfas jóvenes $(97 \%)$ y para ninfas desarrolladas (72\%). Por otra parte Vavrina et al (1995) encontraron que el jabón insecticida M-Pede fue efectivo contra ninfas de primer instar de B. argentifolii

\section{CONCLUSIONES}

Para las condiciones del municipio de Tepetzinlta, Veracruz, las alternativas para el manejo de Bemisia spp. en el cultivo de chile regional (criollo), basadas en aplicaciones de dos veces por semana de los hongos

entomopatógenos B. bassiana, $P$. fumosoroseus, y extractos vegetales y aceites minerales, no solo logran reducir las poblaciones del fitófago en forma sostenida, sino que permiten el logro de producciones de calidad, con altos rendimientos favorables, equivalentes a los del control químico convencional. 


\section{LITERATURA CITADA}

Aguiar, A.; Kass, D.C.; Mora, G. A.; Hilje, L. 2003. Feeding deterrence of three vegetable extracts on Bemisia tabaci adults. Manejo Integrado de Plagas y Agroecología 68:62-70.

Alfaro, M. J. A. 1999. Hongos entomopatógenos contra el pulgón (Myzus persicae Sulzer) en berenjena en Culiacán, Sin. Tesis de Licenciatura de la Facultad de Agronomía de la Universidad Autónoma de Sinaloa, Culiacán, México.

Angulo, B. A.; Traslaviña, R.; Soto, R. L. 1994. Fluctuación del porcentaje de parasitismo en el cultivo de berenjena en el valle de Culiacán, Sin. Memorias del XVII Congreso Nacional de Control Biológico. SMCB-ITAO. 6-7 oct 1994, p. 58-62.

Arcos, C. G.; Hernández, J. H.; Uriza. D. H.; Pozo. O. P.; Olivera, A. O. 1998. Tecnología para producir chile jalapeño en la Planicie Costera del Golfo de México. SAGAR. INIFAP. Centro de Investigaciones Regional Golfo Centro de Investigación Regional Noroeste, 206 pp.

Arcos, C. G.; Hernández, J. H.; Uriza. D. H.; Pozo. O. P.; Olivera, A. O. 1998. Tecnología para producir chile jalapeño en la Planicie Costera del Golfo de México. SAGAR. INIFAP. Centro de Investigaciones Regional Golfo Centro de Investigación Regional Noroeste, 206 pp.

Asaff,T.A.; López, V.E.; Torre, M. de la. 2003. Guerra de insectos y microorganismos: una estrategia natural para el control de plagas. Avance y Perspectivas 21: 291294.

Avilés, G. M. 1999. Mosquita blanca. En Memorias del Curso de Manejo Integrado de Plagas en tomate, chile, pepino y calabaza. Campo Experimental del Valle de Culiacán, Culiacán, Sinaloa.
Avilés, G.M.; Nava, C.U.; Garzón, T.J.; Wong, P.J.J.; Pérez, V.J. 2004. Manejo integrado de la mosquita blanca Bemisia sp. en tomate para consumo en fresco. Centro de Investigación Regional del Noroeste Campo Experimental Valle de Culiacán INIFAP, 76 pp.

Bernal, J.A. 2003. Effect of some natural and synthetic insecticides on Bemisia tabaci

(Gennadius) (Homoptera:Aleyrodidae) in tomato in Panama. Revista de Protección Vegetal 18(1):32-37.

Borad, P.K.; Patel, H.M.; Sisodiya, D.B.; Vaghela, N.M.; Patel, J.R. 2003. Bioefficacy of endosulfan $35 \%$ EC and cypermethrin 5\% EC mixture against insect-pests of cotton H-6. Indian Journal of Entomology 65(3):416-421.

CAB International, 2004. Crop Protection Compendium. Wallingford, UK: $\mathrm{CAB}$ International.

FAO.2004. FAOSTAT Statistical Database 2004. Food and Agricultural Organization of the United Nations, Rome.

Faria, M.; Wraight, S. P.; Naranjo, S. E. 2001. Biological control of Bemisia tabaci with fungi. Crop Protection 20(9)767-778. https://doi.org/10.1016/S0261-2194(01)001 $10-7$

Farman, U.; Baloch, A.F.; Badshah, H. 2006. Estudios de la resistencia de variedades y control químico de la mosquita blanca (Bemisia tabaci Genn.) en algodón. Journal of Biological Sciences 6(2):261264.

https://doi.org/10.3923/jbs.2006.261.264

García F.; Ortega, L.; Guzmán, A. 2000. Influencia de la frecuencia de aplicación de Beaveria bassiana y Paecilomyces fumosoroseus sobre la "Mosquita Blanca" y sus parasitoides. Memorias XXIII Congreso Nacional de Control Biológico. Sociedad Mexicana de Control Biológico. Guanajuato, México, 16-18 de Noviembre del 2000, p.119-122. 
Gindin, G.; Geschtovt, N. U.; Raccah, B.; Barash, I. 2000. Pathogenicity of Verticillium lecanii to different developmental stages of the silverleaf whitefly, Bemisia argentifolii. Phytoparasitica 28(3):27-32 https://doi.org/10.1007/BF02981801

Liu, T.; Stansly, A. P. 1995. Toxicity of biorational insecticides to Bemisia argentifolii (Homoptera: Aleyrodidae) on tomato leaves. J. Econ. Entomol. 88 (3):564-568.

https://doi.org/10.1093/jee/88.3.564

López, M. M. 1998. Alternativas de control para insectos plaga en tomate (Lycopersicon esculentum Mill): Culiacán, Sinaloa. 1995-1996. Tesis de Maestría en Ciencias de la Producción Agrícola. Facultad de Agronomía. Universidad Autónoma de Sinaloa.

Manjula, C.; Nagaraju, C.; Muniyappa, V. 2003. Evaluation of different oil formulations of bio-agent, Beauveria bassiana against different life stages of Bemisia tabaci, the vector of tomato leaf curl virus. Plant Disease Research (Ludhiana) 18(1):25-28.

Mareggiani, G. 2001. Manejo de insectos plagas mediante sustancias semioquímicas de origen vegetal. Manejo Integrado de Plagas (Costa Rica) 60:2230 .

Orozco, S. M.; Farias, L. L.; Pérez, J.; Ramírez, V. N. R. 2000. Uso de Beauveria bassiana para el control de Bemisia argentifolii en melón. Manejo integrado de Plagas (Costa Rica) 56:45-51.

Paula, F.L.; Bleicher, E. 2003. Evaluation of the effect of different types of vegetable oils on whitefly control, on melon. Manejo Integrado de Plagas y Agroecología, 68:53-56.

Piron, M.; Lacordaire, A. I. M. 2006. Lecanicillium lecanii Ve6: insecticida biológico contra Aleyrodidae. Phytoma 591:42-44.
Piron, M.; Lacordaire, A. I. M. 2006.

Lecanicillium lecanii Ve6: insecticida biológico contra Aleyrodidae. Phytoma 591:42-44.

Poprawski, T. J.; Grennberg, S. M.; Ciomperlik, M. 2000. Effect of host plant on Beauveria bassiana and Paecilomyces fumosoroseus induced mortality of

Trialeurodes vaporariorum (Homoptera: Aleyrodidae). Environ. Entomol. 29(5):1048-1053.

https://doi.org/10.1603/0046-225X-29.5.1 048

Prabhaker, N.; Toscano, N. C.; y Coudriet, D. L. 1989. Susceptibility of immature and adult stages of the sweetpotato whitefly (Homoptera: Aleyrodidae) to selected insecticides. J. Econ. Entomol. 82 (4):983-988.

https://doi.org/10.1093/jee/82.4.953

Ramos, E. Q.; Alves, S. B.; Demetrio, C. G. B.; Costa, S. C. 2004. Selección de hongos entomopatógenos para el control de Bemisia tabaci y biotipo B. Manejo Integrado de Plagas y Agroecología 73:21-28.

Ray, A. A. 1982. SAS user's guide. Statistics. SAS Institute Inc. Cary, N.C.

Rodríguez, A. 2003. Una alternativa con Hongos Entomopatógenos nativos para el control de Trialeurodes vaporariorum (West.), en el cultivo de tomate en invernáculos, en Uruguay. Tesis presentada en opción al Grado Científico de Doctor en Ciencias Agrícolas, Universidad Agraria de La Habana, La Habana. Cuba.

Rodríguez, H. C. 2000. Plantas contra plagas. Potencial práctico de ajo, anona, nim, chile y tabaco. RAAPAN. RAAA. México, 133 pp.

Ruiz, V. J.; Bolaños, T. A. 1999. Manejo de Bemisia tabaci mediante barreras vivas $\mathrm{y}$ Paecilomyces en Oaxaca. México. Manejo Integrado de Plagas (Costa Rica) 58:88-96. 
Silva, G.; Lagunes, A.; Rodríguez, J. C.; Rodríguez, D. 2002. Insecticidas vegetales: Vieja-nueva alternativa en el control de plagas. Manejo Integrado de Plagas (CATIE) 61 :25-29.

Valderrábano, J.; Uriarte, J. 2001. Effect of certain Plant extracts on the destruction of eggs and larvae. ITEA $22(1)$ : 179-181. https://doi.org/10.1016/S0367-326X(00)0028 $1-1$

Valle, G. E. do; Lourenção, A. L. ; Novo, J. P. S. 2002. Control químico de huevos y Ninfas de Bemisia tabaco biotipo B (Hemiptera: Aleyrodidae). Scientia Agricola 59(2):291-294.

https://doi.org/10.1590/S0103-9016200200 0200013

Copyright (c) 2015 Alfredo González Acosta, Alfredo G onzález Castro, Roberto A. Mateos Rocha y Julio C. González Cárdenas

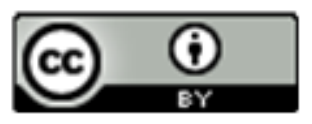

E ste tex to está protegido por una licencia Creative Commons 4.0.

Usted es libre para Compartir —copiar y redistribuir el $m$ aterial en cualquier medio o formato- y Adaptar el documento —remezclar, transformar y crear a partir del material- para cualquier propósito, incluso para fines com erciales, siempre que cumpla la condición de:

Atribución: Usted debe dar crédito a la obra original de manera adecuada, proporcionar un enlace a la licencia, e in dicar si se han realizado cambios. Puede hacerlo en cualquier forma razonable, pero no de forma tal que sugiera que tiene el apoyo del licenciante o 1o recibe por el uso que hace de la obra.

Resumendelicencia - Textocompletocielalicencia 